\title{
Choque circulatorio. Estableciendo metas en la reanimación con líquidos
}

\author{
Silvia del Carmen Carrillo Ramírez, ${ }^{1}$ Pedro Alejandro Elguea Echavarría ${ }^{2}$
}

\section{Resumen}

Se define choque circulatorio como un síndrome precipitado por un colapso sistémico de la perfusión, que resulta en hipoxia celular generalizada y disfunción orgánica múltiple. Este se clasifica en hipovolémico, obstructivo, cardiogénico y distributivo. El tratamiento para cada uno de ellos es específico de la causa que lo precipitó; sin embargo, el punto en el cual coinciden los manejos es la infusión de líquidos. Cabe mencionar que administrar una cantidad inadecuada, ya sea por déficit o exceso, tiene efectos deletéreos en el paciente, por lo que es de suma importancia conocer los principios a seguir para guiar la administración de soluciones. El objetivo de la presente revisión es plantear de una manera ordenada las metas que se deben fijar para evitar la sobrecarga de líquidos. Para esto, se clasifica la reanimación del individuo en estado de choque en cuatro etapas que serán revisadas con detalle.

Palabras clave: Choque circulatorio, infusión de líquido, reanimación con líquido.

\section{INTRODUCCIÓN}

Se ha establecido que el estado de choque corresponde a casi dos tercios de los casos que son tratados en las áreas críticas, ya sea en el ámbito prehospitalario, las salas de

\footnotetext{
${ }^{1}$ Servicio de Terapia Intensiva.

2 Residente del segundo año de la especialidad.
}

Medicina del Enfermo Adulto en Estado Crítico. Hospital Ángeles Pedregal.

Correspondencia:

Dr. Pedro Alejandro Elguea Echavarría

Correo electrónico: alejandro_elguea@hotmail.com

Aceptado: 22-09-2016.

Este artículo puede ser consultado en versión completa en http:// www.medigraphic.com/actamedica

\section{Summary}

Circulatory shock is defined as a syndrome precipitated by a systemic collapse of the perfusion, resulting in widespread cell hypoxia and multiple organ dysfunction. It is classified into hypovolemic, obstructive, cardiogenic and distributive. The point at which the management of each of them coincides is the infusion of fluids. It is important to mention that an inadequate quantity of fluids, either deficient or excessive, has deleterious effects on the patient; therefore, it is extremely important to know the principles to guide the management of shock. The aim of this review is to present in an orderly manner the goals that must be set to avoid fluid overload. To achieve this, fluid resuscitation is classified into four stages that will be reviewed in detail.

Key words: Circulatory shock, infusion of fluid, fluid resuscitation.

emergencias o las unidades de cuidados intensivos. Éste se define como un síndrome precipitado por colapso sistémico de la perfusión, que resulta en hipoxia celular generalizada y disfunción orgánica múltiple. ${ }^{1}$

El diagnóstico se establece ante la presencia de alguno de los siguientes criterios: ${ }^{2}$

A) Características clínicas: se cuenta con tres ventanas: renal (oliguria), cutánea (piel fría, pálida y diaforética) y neurológica (confusión, desorientación y somnolencia).

B) Características hemodinámicas: la taquicardia e hipotensión son datos francos de choque, pero dependen del tipo de choque que se presente, así como de la etapa. Además, los cambios en la tensión arterial también dependen de las características del sujeto; por ejemplo, si tiene el antecedente de hipertensión arterial sistémica.

C) Características bioquímicas: hiperlactatemia mayor a $2 \mathrm{mmol} /$ litro. 
Dado que el estado de choque es siempre una emergencia, el diagnóstico y el tratamiento van de la mano.

\section{PRIORIDADES EN EL MANEJO INICIAL}

El manejo del estado de choque se centra de manera inicial en inhibir la causa que lo produjo: controlar la hemorragia en la hipovolemia, dar antibióticos en la sepsis, descomprimir un neumotórax a tensión en el choque obstructivo, angioplastia coronaria percutánea o trombólisis en el caso de infarto agudo de miocardio como causa de choque cardiogénico. ${ }^{3}$

De cualquier manera, hay un punto en el cual coinciden los manejos de la persona en choque: la infusión de líquidos. Esta intervención parece sencilla y lógica; sin embargo, debemos ser cautelosos, ya que existen dos extremos en los que se puede caer: el déficit y el exceso.

El déficit se traduce en persistencia de la hipovolemia, por lo que el volumen sanguíneo circulante efectivo se concentra en los órganos vitales, disminuyendo la perfusión a la piel, el intestino y el riñón. Lo anterior desencadena una respuesta neurológica simpática y activa el sistema renina-angiotensina-aldosterona. La respuesta inflamatoria sistémica se intensifica. Clínicamente, ante la falta de líquidos, se utilizan de manera inadecuada aminas vasoactivas que incrementan la vasoconstricción y, por lo tanto, la hipoperfusión e isquemia. Esto se traduce en persistencia del choque, hipoperfusión, falla renal y aumento de la mortalidad. ${ }^{4}$

Por otro lado, el exceso de líquidos nos lleva a sobrecarga, complicación relativamente frecuente en los pacientes críticos que, además, es consecuencia del mismo tratamiento. Lo anterior conduce a un incremento de la presión venosa, fuga a tercer espacio y repercusión en cada uno de los órganos y sistemas por persistencia de hipoxia tisular, como se establece en el cuadro 1. Cabe mencionar que son muchos los estudios en los que ya se ha demostrado que la sobrecarga de volumen repercute de manera significativa en el aumento de la mortalidad de los individuos. ${ }^{5}$

De esta manera, es de suma importancia encontrar el punto medio en que logremos el equilibrio hídrico y, por lo tanto, hemodinámico. Para esto es importante plantear las metas correctas en el momento indicado. Actualmente se recomienda dividir en cuatro etapas la reanimación del paciente crítico: salvamento, optimización, estabilización y desreanimación. ${ }^{6}$ A continuación, detallaremos cada una de ellas:

\section{ETAPA DE SALVAMENTO}

En este momento, nos enfocaremos en mantener vivo al sujeto, planteando como meta estabilizar las funciones y constantes vitales. Por años, el manejo inicial de la persona grave se ha centrado en el manejo del $\mathrm{ABC}$ :

A: Abrir y mantener una vía aérea permeable, realizando intubación endotraqueal si así lo requiere el individuo; hay que recordar que el estado de choque es una indicación para realizar este procedimiento. Además, la ventilación mecánica invasiva tiene el beneficio de disminuir la demanda de oxígeno por los músculos res-

\begin{tabular}{|c|c|c|}
\hline Órgano o sistema & Efecto & Manifestación clínica \\
\hline Sistema nervioso central & Edema cerebral & Estado neurológico alterado. Delirium \\
\hline Sistema respiratorio & $\begin{array}{l}\text { Edema pulmonar, } \\
\text { derrame pleural }\end{array}$ & $\begin{array}{l}\text { Incremento del trabajo respiratorio. Resistencia } \\
\text { en el intercambio de gases. Reducción de la } \\
\text { distensibilidad pulmonar. Acumulación } \\
\text { de agua pulmonar extravascular }\end{array}$ \\
\hline Sistema cardiovascular & $\begin{array}{l}\text { Edema de miocardio, } \\
\text { derrame pleural }\end{array}$ & $\begin{array}{l}\text { Alteración de la contractilidad. Disfunción diastólica. } \\
\text { Trastornos de conducción }\end{array}$ \\
\hline Sistema gastrointestinal & Edema intestinal, ascitis & $\begin{array}{l}\text { Mala absorción. Íleo. Translocación bacteriana. } \\
\text { Hipertensión intraabdominal }\end{array}$ \\
\hline Sistema hepatobiliar & Congestión hepática & Colelitiasis. Función de síntesis biliar alterada \\
\hline Sistema renal & $\begin{array}{l}\text { Edema intersticial renal, } \\
\text { presión venosa renal elevada }\end{array}$ & $\begin{array}{l}\text { Lesión renal aguda. Uremia. Retención } \\
\text { de agua y sodio }\end{array}$ \\
\hline $\begin{array}{l}\text { Piel y sistema } \\
\text { musculoesquelético }\end{array}$ & $\begin{array}{l}\text { Edema tisular, drenaje } \\
\text { linfático alterado, alteración } \\
\text { de la microcirculación }\end{array}$ & $\begin{array}{l}\text { Pobre recuperación de heridas. Úlceras } \\
\text { por presión. Infección de heridas }\end{array}$ \\
\hline Sistema hematológico & Hemodilución & $\begin{array}{l}\text { Persistencia del sangrado debido } \\
\text { a coagulopatía por dilución }\end{array}$ \\
\hline
\end{tabular}


piratorios y reducir la postcarga del ventrículo izquierdo al incrementar la presión intratorácica. ${ }^{7}$ Se debe tener mucha precaución con la sedación, ya que los fármacos que se usan con mayor frecuencia (midazolam, propofol) se asocian a una baja de la presión arterial y el gasto cardiaco.

B: Ventilar y mantener una apropiada oxigenación, asegurándonos mediante auscultación pulmonar de que el aire entra adecuadamente a los pulmones. De preferencia, se debe confirmar la correcta intubación mediante capnografía, ya que es el estándar de oro. Una vez confirmada la idónea posición del tubo, se debe mantener $\mathrm{SpO}_{2}$ superior al 94\%, aunque sea necesario administrar una $\mathrm{FiO}_{2}$ al $100 \%$. En caso de que exista alguna complicación respiratoria, esta debe ser resuelta, ya sea administrando broncodilatadores para solucionar la presencia de espasmo, diuréticos en caso de edema pulmonar, etcétera. ${ }^{8}$

C: Circulación. El objetivo principal será estabilizar la tensión arterial media. Fisiológicamente, se acepta una TAM superior a $65 \mathrm{mmHg}$, ya que este es el punto en el que la mayoría de los lechos vasculares pierden su capacidad de autorregulación local. Esta recomendación se basa en un estudio clínico aleatorizado reciente en el que se comparó el efecto de mantener una TAM de 80 a $85 \mathrm{mmHg}$ contra una de 75 a $70 \mathrm{mmHg}$ en sujetos con choque séptico. No se encontraron diferencias significativas en la mortalidad a los 28 o 90 días entre los dos grupos. ${ }^{9}$

La cifra también depende del caso que se esté atendiendo, así como de la persona. Una complicación seria de la reanimación en el paciente con choque hemorrágico es la coagulopatía inducida por trauma, en la que la administración agresiva de soluciones cristaloides induce dilución de los factores de coagulación, lo que se traduce en persistencia de la hemorragia y, por tanto, aumento de la mortalidad. Para evitar esta complicación se recomienda utilizar la hipotensión permisiva —en la que la meta al infundir soluciones cristaloides es una tensión arterial sistólica de $90 \mathrm{mmHg}$ o una TAM de $40 \mathrm{mmHg}$ - hasta que sea posible iniciar la transfusión de hemoderivados, incluyendo paquetes globulares, plasma y crioprecipitados, o hasta lograr el control de la hemorragia. ${ }^{10}$

Para estabilizar la TAM, la infusión de líquidos debe ser agresiva, en promedio 20 a $30 \mathrm{~mL} / \mathrm{kg} / \mathrm{h}$. Esto aplica principalmente en caso de choque hipovolémico y choque distributivo, pero incluso el individuo con choque cardiogénico se beneficia de líquidos, ya que la presencia de edema agudo pulmonar puede resultar en disminución del volumen intravascular efectivo y presentar un componente de hipovolemia relativa. Por supuesto, la reanimación debe ser cautelosa, recordando que normalmente el choque cardiogénico se asocia a disminución de la función sistólica y aumento de la precarga, por lo que la sobrecarga de líquidos ocurre con mayor facilidad y esto repercute en el pronóstico del sujeto. ${ }^{11}$

En caso de choque obstructivo, la cantidad de líquidos es menor, ya que al resolver el problema (descompresión de un neumotórax, pericardiocentesis) la TAM mejora de forma importante. ${ }^{12}$

En cuanto a la velocidad de infusión, no existen reglas. Tanto la cantidad como la velocidad deben ser ajustadas a cada persona, valorando el incremento de la tensión arterial, la disminución de la frecuencia cardiaca, el aumento en la diuresis o la subida paulatina en el volumen sistólico si se cuenta con dispositivo de monitoreo hemodinámico mínimamente invasivo. El edema pulmonar es la complicación más grave de la administración de líquidos, por lo que es importante auscultar al paciente constantemente. Para evitar la sobrecarga en esta etapa de la reanimación, se aconseja administrar cargas de 300 a 500 mL cada 20 a 30 minutos y valorar el resultado, vigilando que no se presenten complicaciones. ${ }^{13}$

El tipo de solución a utilizar es un tema amplio, por lo que no se abordará en este artículo.

Cuando la hipotensión es severa o no responde a la infusión de líquidos, será necesario iniciar infusión de aminas vasoactivas, procurando discontinuarlas cuando la cantidad de líquidos haya alcanzado el requerimiento y la hipovolemia se haya corregido. No existe consenso del tiempo óptimo para iniciar aminas vasoactivas en esta etapa; sin embargo, se debe hacer con precaución y, de preferencia, bajo monitoreo hemodinámico del gasto cardiaco, ya que se ha comprobado en estudios clínicos que la norepinefrina puede incrementar o disminuir el gasto cardiaco, y esto depende del balance de volumen reclutado (incremento de la presión media de llenado sistémico), cambio en la resistencia al retorno venoso y la función cardiaca de base. ${ }^{14}$ Por este motivo, es de mucha utilidad contar con monitoreo hemodinámico mínimamente invasivo antes de iniciar agentes vasoactivos.

Por otro lado, el iniciar aminas con persistencia de hipovolemia empeorará la perfusión tisular periférica por aumento de la vasoconstricción. En un estudio retrospectivo con 2,849 individuos con choque séptico se examinó la influencia del inicio de líquidos y agentes vasoactivos, así como del total de volumen infundido sobre la mortalidad hospitalaria. Una de las conclusiones fue que iniciar aminas dentro de la primera hora de la reanimación puede ser deletéreo para el sujeto. ${ }^{15}$

Los agentes vasoactivos que se usan con mayor frecuencia son norepinefrina y dopamina. Sin embargo, cabe mencio- 
nar que se ha documentado un incremento de la mortalidad con la utilización de la dopamina, por lo que el empleo de norepinefrina a dosis de 0.1 a $2.0 \mu \mathrm{g} / \mathrm{kg} / \mathrm{min}$ se ha convertido en la primera elección. Además, la norepinefrina también posee efecto betaadrenérgico, por lo que ejerce inotropismo positivo a la vez, compensando en cierta manera el aumento de la postcarga debido a vasoconstricción. ${ }^{16}$

Por otro lado, se ha documentado que se puede presentar deficiencia de vasopresina en personas con estados hiperdinámicos en choque distributivo, por lo que la infusión de vasopresina en dosis no mayores a 0.04 u por minuto se asocia a incremento de la tensión arterial y se recomienda como el vasopresor de segunda elección. ${ }^{17}$

El uso de inotrópicos como dobutamina o levosimendán debe quedar restringido sólo en caso de encontrar evidencia de disfunción miocárdica o bajo gasto cardiaco refractario a líquidos y aminas vasoactivas. Es raro que se usen en esta etapa de salvamento debido a que se requieren más datos clínicos y hemodinámicos. ${ }^{18}$

\section{ETAPA DE OPTIMIZACIÓN}

En este momento, ya se ha estabilizado la función ventilatoria y circulatoria, y obtenido una tensión arterial en meta. A continuación, se utiliza un monitoreo más profundo, en el que el objetivo es optimizar la adecuada distribución y entrega de oxígeno, vigilando los niveles de lactato y variables de oxigenación.

\section{Lactato}

Esta sustancia está bien establecida para la detección de condiciones anaeróbicas, ya que es el producto final de la glucólisis anaerobia. Más que una medida diagnóstica en choque, se ha establecido como un valor pronóstico. Un valor mayor a $4 \mathrm{mmol} /$ litro se ha asociado en estudios clínicos a incremento de la mortalidad. ${ }^{19}$ Asimismo, el aclaramiento de lactato es de suma importancia. Un aclaramiento de lactato mayor al $10 \%$ en las primeras seis horas se asocia a incremento en la supervivencia. ${ }^{20}$ De la misma manera ocurre cuando el lactato se logra llevar a valores normales en las primeras 24 a 48 horas. $^{21}$

\section{Marcadores de oxigenación y gasto cardiaco}

La saturación venosa mixta de oxígeno $\left(\mathrm{SvO}_{2}\right)$ obtenida de la arteria pulmonar representa probablemente el mejor indicador de la adecuación del transporte de oxígeno $\left(\mathrm{DO}_{2}\right)$. Sin embargo, también la saturación venosa central de oxígeno $\left(\mathrm{SvCO}_{2}\right)$, obtenida en la aurícula derecha, ha demostrado una buena correlación con la $\mathrm{SvO}_{2}$ (aunque sobreestima en torno al 5\%). ${ }^{22}$
La interpretación de la $\mathrm{SvcO}_{2}$ se realiza de la siguiente manera:

$\mathrm{SvO}_{2} 65 \%$ a $75 \%=$ Normal.

$\mathrm{SvO}_{2}<65 \%=$ Indica disminución en la disponibilidad de oxígeno (usualmente debido a anemia o un bajo gasto cardiaco).

$\mathrm{SvO}_{2}$ cerca del $50 \%$ = Indica oxigenación tisular alterada.

$\mathrm{SvO}_{2}>75 \%=$ Indica alteración en el consumo de oxígeno, que usualmente es el resultado de daño celular en la sepsis severa o choque séptico.

Asimismo, una diferencia arteriovenosa mayor al 50\% representa hipoxia celular $\left(\mathrm{SaO}_{2}-\mathrm{SvO}_{2}\right)$. Otra herramienta útil para detectar hipoperfusión tisular es la diferencia arteriovenosa de $\mathrm{CO}_{2}$. Diferentes trabajos han correlacionado inversamente su valor a los valores de índice cardiaco. Niveles de $\mathrm{P}(\mathrm{v}-\mathrm{a}) \mathrm{CO}_{2}>6 \mathrm{mmHg}$ han demostrado ser útiles en la detección de hipoperfusión persistente a pesar de la normalización de la $\mathrm{SvCO}_{2}{ }^{23}$

Cuando una de estas variables persiste alterada o existe sospecha clínica o hemodinámica de que aún existe hipovolemia y/o hipoperfusión tisular, es el momento de realizar un reto de líquidos. Para ello, primero hay que determinar si el paciente tiene o no un déficit de líquidos. Para esto existen variables hemodinámicas que ayudan a predecir si el individuo es potencialmente respondedor a líquidos; entre ellas se encuentran la variabilidad de volumen sistólico $>10 \%$ y la variabilidad de presión de pulso $>13 \%$. Otra maniobra recomendada es el levantamiento de piernas, en el cual, a través de monitoreo hemodinámico mínimamente invasivo, se vigila si existe incremento del volumen sistólico al elevar las piernas $45^{\circ} .{ }^{24}$

Una vez que se ha verificado que el sujeto es potencial respondedor a líquidos, se recomienda administrar una carga de $250 \mathrm{~cm}^{3}$ en dos a cinco minutos. Posteriormente a esto, se valora de nuevo el volumen sistólico. Una respuesta positiva a la administración de líquidos se considera un incremento del volumen sistólico en 10 a 15\%. En caso de que la respuesta sea positiva, se debe volver a realizar el test hasta que la prueba sea negativa. A esto se le denomina "maximización del volumen sistólico". ${ }^{25}$

De esta manera, se considera seguro administrar líquidos en cargas parciales para valorar sus efectos. Esta medida se considera eficaz para prevenir la sobrecarga de líquidos, que como ya hemos mencionado, aumenta la mortalidad del paciente crítico. ${ }^{26}$

\section{ETAPA DE ESTABILIZACIÓN}

En esta etapa se considera que la persona ya cuenta con una adecuada perfusión tisular aunque aún requiera de so- 
porte hemodinámico con aminas vasoactivas. Usualmente no se requieren líquidos de mantenimiento, ya que con frecuencia es suficiente con nutrición enteral o parenteral más medicamentos. En caso de que se tenga sospecha clínica de déficit de líquidos, se puede realizar una vez más un test de líquidos para lograr suspender los vasopresores.

\section{ETAPA DE EVACUACIÓN}

El objetivo de esta etapa es eliminar el exceso de líquidos en los individuos que se encuentran hemodinámicamente estables y no tienen evidencia de hipoperfusión tisular. No existe consenso acerca de en qué momento iniciar la excreción de líquidos. Sin embargo, se sugiere considerar la restricción del aporte en aquellos sujetos que han logrado mantenerse hemodinámicamente estables, y considerar el apoyo con diurético para lograr un balance negativo en quienes no son capaces de eliminar líquidos de forma espontánea.

Se publicó un estudio que compara distintos métodos para realizar esta tarea enfocada a la persona con síndrome de distrés respiratorio agudo. En él se recomienda la administración de diurético en quienes han cumplido más de 12 horas sin aminas vasoactivas, cuentan con TAM mayor a $60 \mathrm{mmHg}$, con la meta de lograr una presión venosa central de $4 \mathrm{mmHg}$ y/o una presión de oclusión de la arteria pulmonar menor a $8 \mathrm{mmHg}$, repitiendo la administración de diurético siempre y cuando tengan una apropiada respuesta, con adecuado gasto urinario. Se concluyó que con este esquema se logró un mejor balance de líquidos comparado con otros esquemas. ${ }^{26}$

\section{CONCLUSIÓN}

La reanimación con líquidos es una de las intervenciones realizadas con mayor frecuencia en salas de emergencia y unidades de terapia intensiva; sin embargo, si no se lleva a cabo de manera adecuada, puede incluso incrementar el daño sobre el individuo. Por ello, es de suma importancia establecer prioridades en distintos momentos de acuerdo a la situación del sujeto, para administrar líquidos de forma concientizada, con bases y objetivos establecidos, y mejorar el pronóstico de los pacientes que atendemos día a día.

\section{REFERENCIAS}

1. Parrillo J, Dellinger P. Critical care medicine. Principles of diagnosis and management in the adult. Chapter 21; Circulatory shock. 4th ed. Philadelphia, USA: Elsevier Saunders; 2014. p. 303.

2. Vincent JL, Ince C, Bakker J. Circulatory shock —an update: a tribute to Professor Max Henry Weil. Cit Care. 2012; 16: 239

3. Mesquida J, Borrat X, Lorente JA, Masipa J, Baigorria F. Objetivos de la reanimación hemodinámica. Med Intensiva. 2011; 35 (8): 499-508.

4. Joosten A, Alexander B, Cannesson M. Defining goals of resuscitation in the critically ill patient. Crit Care Clin. 2015; 31: 113-132.
5. Ogbu OC, Murphy DJ, Martin GS. How to avoid fluid overload. Curr Opin Crit Care. 2015; 2 (4): 315-321.

6. Vincent JL, De Backer D. Circulatory shock. N Engl J Med. 2013; 369: 1726-1734.

7. Singh I, Pinsky MR. Heart-lung interactions. In: Papadakos PJ, Lachmann B, eds. Mechanical ventilation: clinical applications and pathophysiology. Philadelphia: Saunders Elsevier; 2008. pp. 173-184.

8. Wacker D, Winters M. Shock. Emerg Med Clin N Am. 2014; 32: 747-758.

9. Asfar P, Teboul JL, Radermacher P. High versus low blood-pressure target in septic shock. N Engl J Med. 2014; 371: 283-284.

10. Egea-Guerrero JJ, Freire AM, Serrano LA, Quintana DM et al. Objetivos y nuevas estrategias de resucitación en el paciente traumatizado grave. Med Intensiva. 2014; 38 (8): 502-512.

11. Klein T, Ramani GV. Assessment and management of cardiogenic shock in the emergency department. Cardiol Clin. 2012; 30 (4): 651-664.

12. Spodick DH. Acute cardiac tamponade. N Engl J Med. 2003; 349: 684-690.

13. Dellinger RP, Levy MM, Rhodes A, Annane D, Gerlach H, Opal SM et al. Surviving Sepsis Campaign: international guidelines for management of severe sepsis and septic shock: 2012. Crit Care Med. 2013; 41: 580-637.

14. Maas JJ, Pinsky MR, de Wilde RB. Cardiac output response to norepinephrine in postoperative cardiac surgery patients: Interpretation with venous return and cardiac function curves. Crit Care Med. 2013; 41: 143-150.

15. Waechter J, Kumar A, Lapinsky SE, Marshall J, Dodek P, Arabi Y et al. Interaction between fluids and vasoactive agents on mortality in septic shock: a multicenter, observational study. Crit Care Med. 2014; 42: 2158-2168.

16. De Backer D, Biston P, Devriendt J, Madl C, Chochrad D, Aldecoa C et al, SOAP II Investigators: Comparison of dopamine and norepinephrine in the treatment of shock. N Engl J Med. 2010; 362: 779-789.

17. Russell JA, Walley KR, Singer J, Gordon AC, Hébert PC, Cooper DJ et al. Vasopressin versus norepinephrine infusion in patients with septic shock. N Engl J Med. 2008; 358 (9): 877-887.

18. Kanter J, DeBlieux P. Pressors and inotropes. Emerg Med Clin North Am. 2014; 32 (4): 823-834.

19. Trzeciak S, Dellinger RP, Chansky ME, Arnold RC, Schorr C, Milcarek $B$ et al. Serum lactate as a predictor of mortality in patients with infection. Intensive Care Med. 2007; 33 (6): 970-977.

20. McNelis J, Marini CP, Jurkiewicz A, Szomstein S, Simms HH, Ritter G et al. Prolonged lactate clearance is associated with increased mortality in the surgical intensive care unit. Am J Surg. 2001; 182 (5): 481-485.

21. Nguyen HB, Rivers EP, Knoblich BP, Jacobsen G, Muzzin A, Ressler JA et al. Early lactate clearance is associated with improved outcome in severe sepsis and septic shock. Crit Care Med. 2004; 32 (8): 1637-1642.

22. Reinhart K, Kuhn HJ, Hartog C, Bredle DL. Continuous central venous and pulmonary artery oxygen saturation monitoring in the critically ill. Intensive Care Med. 2004; 30: 1572-1578.

23. Vallée F, Vallet B, Mathe O, Parraguette J, Mari A, Silva S et al. Central venous to arterial carbon dioxide difference: an additional target for goal directed therapy in septic shock. Intensive Care Med. 2008; 34: 2218-2225.

24. Ochagavíaa A, Baigorria F, Mesquidaa J, Ayuelab JM, Ferrándizc A, Garcíaa X et al. Grupo de Trabajo de Cuidados Intensivos Cardiológicos y RCP de la SEMICYUC. Monitorización hemodinámica en el paciente crítico. Recomendaciones del Grupo de Trabajo de Cuidados Intensivos Cardiológicos y RCP de la Sociedad Española de Medicina Intensiva, Crítica y Unidades Coronarias. Med Intensiva. 2014; 38: 154-169.

25. Cecconi M, Parsons AK, Rhodes A. What is a fluid challenge? Curr Opin Crit Care. 2011; 17: 290-295.

26. Grissom CK, Hirshberg EL, Dickerson JB, Brown SM, Lanspa MJ, Liu KD et al. Fluid management with a simplified conservative protocol for the acute respiratory distress syndrome. Crit Care Med. 2015; 43 (2): 288-295. 